\title{
Consistent formulation of the power-law rhe- ology and its application to the spreading of non-Newtonian droplets
}

\author{
L. Devaud, M. Sellier and A.-R. Al-Behadili
}

Keywords. Non-Newtonian, Wetting, Ostwald power-law, Power-law.

\begin{abstract}
In this work, we introduce a general form of the Navier-Stokes equations for Generalized Newtonian fluids with an Ostwald power-law. The derivation, based on the covariant formalism, is frame-independent and gives rise to a source term in the Navier-Stokes equations referred to as the Ostwald vector which is characterized by the power-law exponent. The governing equations are then simplified in the long-wave approximation framework and applied to the spreading of an axisymmetric gravity current in the creeping flow regime. Well-known spreading laws are recovered through similarity solutions and a new derivation based on scaling arguments is proposed. Experimental results related to the spreading of gravity current are then presented and the potential to infer unknown rheological parameters from spreading rates is critically discussed in the context of a thorough error analysis.
\end{abstract}

\section{Introduction}

Non-Newtonian fluids appear frequently in our daily life. Unfortunately, the dependence of their viscosity on the applied stress makes them hard to model theoretically. Understanding their behaviour is important for industrial and experimental applications. Up to now, a widely used and accepted way to describe the non-Newtonian response to an applied stress is using the Ostwald power-law which is applicable for Generalized Newtonian fluids. However, a simpler form of this power-law is more commonly used, writing $\zeta=K \dot{\gamma}^{n}$, where $\zeta$ is the shear stress, $\dot{\gamma}$ the shear rate and $K$ the consistency index (see [13] for example) irrespectively of the geometry considered and the corresponding coordinate system. The purpose of the present work is to derive, using and justifying the Ostwald power-law, an equivalent form of the Navier-Stokes equation for fluids with a power-law rheology using the 
covariance formalism. Hence, the resulting equations are valid irrespective of the coordinate system. After establishing the general equivalent form of the Navier-Stokes equation for Ostwald power-law fluids, our aim is to apply it for the study of droplets spreading under the action of gravity and to investigate the effect of the non-Newtonian rheology on the spreading radius variation. Some experimental results will be presented in order to support the theoretical approach.

The spreading of non-Newtonian droplets has attracted much attention in the past because of its prevalence in a number of contexts such as the spreading of pesticide on leaves, inkjet printers, or the spreading of geophysical fluids. Much insight was made possible by the seminal work of Gratton et al. [7] who derived a similarity solution for the axisymmetric spreading of a gravity current of a power-law fluid. The analysis was later extended by Starov et al. [19] to include the effect of capillarity. Rafaï et al. [15] studied the spreading of non-Newtonian droplets experimentally and concluded that the 1/10 exponent of Tanner's law [20] describing the evolution of the spread radius was remarkably robust. Ahmed et al. [1] solved numerically the two-dimensional form of the lubrication approximation with a power-law rheology to study the spreading and sliding of a non-Newtonian droplet down an inclined plane. Recently, the effect of a more complex rheology on the wetting dynamics has been investigated. Balmforth et al. [2] studied the effect of viscoplasticity using the Herschel-Bulkley constitutive law while Uppal et al. included the effect of thixotropy in the spreading dynamics [21].

The ability to correctly predict the spreading of droplets or gravity currents opens up the opportunity to indirectly infer the viscosity or the rheology of the fluid from spreading rate observations. This is important when the fluid is not directly accessible for safety reason. For example, Foit [6] envisaged to use the spreading laws of isothermal and non-isothermal gravity current to deduce the viscosity of radioactive melts during a hypothetical core meltdown. In the food industry, the consistency of food product is often assessed using a Botswick consistometer, the idea of which is simply to let a fix volume of fluid spread on a surface and monitor its spreading rate. Corresponding theoretical models were developed in $[14,13,2]$. In a related study, Sellier et al. [18] deduced the viscosity of aerosol matter by considering the rate of closure of a dry hole poked in a sessile droplet of that matter. The spreading of a power-law gravity current was recently studied by Sayag and Worster [16] and Longo et al. [11] for the constant volume or constant flux release. The authors demonstrate that the power-law rheological parameters can in principle be deduced from spreading experiments but more reliably/generally so in the constant flux case.

The main purpose of this paper is to introduce a general form of the Navier-Stokes equations for Generalized Newtonian fluids with an Ostwald power-law. This general form is then reduced to the required form to describe the spreading of a power-law droplet. The paper then describes in detail the 
derivation of a similarity solution for the governing equations. This derivation is supported by a scaling law analysis which support the spreading laws predicted by the similarity solutions. Experimental data on the spreading of different liquids are finally presented and discussed in the context of the spreading laws earlier derived.

\section{Non-Newtonian fluids and the Ostwald power-law}

\subsection{Covariance formalism}

Covariance is a very useful concept. Being able to change easily the working coordinates is all the more important in fluid mechanics where the common flow domains are conveniently described by varied coordinate systems: Cartesian, cylindrical or spherical. Writing equations in a covariant form makes them independent of the working coordinate system. The equation of momentum conservation is [8]

$$
\rho \frac{D \vec{v}}{D t}=\overrightarrow{f_{V}}+\operatorname{div}[\sigma]
$$

where $\overrightarrow{f_{V}}$ represents the volume forces and $[\sigma]$ the stress tensor. The first step in order to work with covariant equations is to write $[\sigma]$ in the correct tensorial form. The covariant form of the stress tensor's $(i, j)$ component for a Newtonian fluids is

$$
\sigma^{i j}=\left(-p+\xi D_{k} v^{k}\right) g^{i j}+2 \eta S^{i j}
$$

where $[g]$ is the space metric tensor, $D_{i}$ is the covariant derivative given by $D_{i} v^{j}=\partial_{i} v^{j}+v^{k} \Gamma_{i k}^{j}\left(\Gamma_{i k}^{j}\right.$ being the Christoffel symbol of the $\left.2^{\text {nd }} k i n d\right)$ and $S^{i j}=\frac{1}{2}\left(g^{i k} D_{k} v^{j}+g^{j k} D_{k} v^{i}\right)$. The idea of the covariant derivation is to consider both the variation of the component (expressed in the partial derivatives) and the variations of the basis vectors themselves (expressed in the Christoffel terms), see [5]. The Christoffel symbols can easily be calculated for cylindrical and spherical coordinates and they all are equal to zero for Cartesian coordinates.

\subsection{Ostwald power-law}

In order to describe the behaviour of non-Newtonian fluids, Ostwald proposed a phenomenological law. He designed his law to be a generalization of the Newtonian behaviour (the law is applied for the so-called Generalized Newtonian fluids). Ostwald looked for a simple, tensorial law. In order to keep a tensorial aspect, he used the tensorial components of the strain rate tensor. The pre-factor he added to the tensor needed to be a scalar because of the isotropy of the fluid. To keep the tensorial aspect of the law, the pre-factor had to be an invariant of the strain rate tensor. The first invariant, the trace, is zero in the case of incompressible flows. The third one, the determinant, is 
not quadratic and is zero in the case of a planar constraint ${ }^{1}$. The most simple invariant to use is thus the second one, $\frac{1}{2}\left(\operatorname{Tr}[S]^{2}-\operatorname{Tr}\left[S^{2}\right]\right)$.

At this point, Ostwald could have chosen either a power law or a polynomial law to describe the stress of non-Newtonian fluids. The power law has the advantage that the exponent may be non-integer. He chose the latter, and designed his law in order to have $n=1$ for Newtonian fluids. For an incompressible flow, the Ostwald law in covariant form is:

$$
\sigma^{i j}=-p g^{i j}+2 K\left(2 S_{l k} S^{k l}\right)^{\frac{n-1}{2}} S^{i j}=-p g^{i j}+2 \frac{K}{\tau^{n-1}}\left(2 \tau^{2} S_{l k} S^{k l}\right)^{\frac{n-1}{2}} S^{i j},
$$

where we have introduced the characteristic time $\tau$ in order to make $2 S_{l k} S^{k l}$ dimensionless. Henceforth, we will refer to the scalar pre-factor $2 \tau^{2} S_{l k} S^{k l}$ as $\alpha$ (which is dimensionless) ${ }^{2}$ and to $\frac{K}{\tau^{n-1}}$ as $\eta_{0}$ which has the dimension of a viscosity. Another explanation of this law can be found in [17] where Schowalter uses linear algebra in order to justify the law; unfortunately he comes to the conclusion that the Ostwald-de Waele model is "in fact not a law at all, but an attempt to find an empirical curve fitting with the maximum of simplicity".

\subsection{Generalized Navier-Stokes equation}

In the framework of the Ostwald power-law and in covariant formalism, the stress tensor for an incompressible flow is thus given by (see eq. (3))

$$
\sigma^{i j}=-p g^{i j}+2 \eta_{0} \alpha^{\frac{n-1}{2}} S^{i j},
$$

Let us recall that $S^{i j}=\frac{1}{2}\left(g^{i r} D_{r} v^{j}+g^{j r} D_{r} v^{i}\right)$ and $\alpha=2 \tau^{2} S_{l k} S^{k l}$. Taking the divergence of the tensor, we get:

$$
\begin{aligned}
(\operatorname{Div}[\sigma])^{i} & =-D_{j} p g^{i j}+2 \eta_{0} D_{j}\left(\alpha^{\frac{n-1}{2}} S^{i j}\right) \\
& =-(G \overrightarrow{r a d} p)^{i}+2 \eta_{0} \alpha^{\frac{n-1}{2}} D_{j}\left(S^{i j}\right)+2 \eta_{0} D_{j}\left(\alpha^{\frac{n-1}{2}}\right) S^{i j} \\
& =-(G \overrightarrow{r a d p})^{i}+\eta_{0} \alpha^{\frac{n-1}{2}} D_{j}\left(g^{i r} D_{r} v^{j}+g^{j r} D_{r} v^{i}\right)+\eta_{0}(n-1) \alpha^{\frac{n-3}{2}} D_{j}(\alpha) S^{i j}
\end{aligned}
$$

But because the metrics covariant derivative is zero, the above can be rewritten as:

$$
=-(G \overrightarrow{r a d} p)^{i}+\eta_{0} \alpha^{\frac{n-1}{2}} g^{i r} D_{j} D_{r} v^{j}+\eta_{0} \alpha^{\frac{n-1}{2}} g^{j r} D_{j} D_{r} v^{i}+\eta_{0}(n-1) \alpha^{\frac{n-3}{2}} D_{j}(\alpha) S^{i j}
$$

And since the covariant derivatives commute in flat space, we have:

$$
\begin{aligned}
& =-(G \overrightarrow{r a d p})^{i}+\eta_{0} \alpha^{\frac{n-1}{2}} g^{i r} D_{r}(D i v \vec{v})+\eta_{0} \alpha^{\frac{n-1}{2}}(\Delta \vec{v})^{i}+\eta_{0}(n-1) \alpha^{\frac{n-3}{2}} D_{j}(\alpha) S^{i j} \\
& =-(G \overrightarrow{r a d} p)^{i}+\eta_{0} \alpha^{\frac{n-1}{2}}(G \overrightarrow{r a d}(D i v \vec{v}))^{i}+\eta_{0} \alpha^{\frac{n-1}{2}}(\Delta \vec{v})^{i}+\eta_{0}(n-1) \alpha^{\frac{n-3}{2}} D_{j}(\alpha) S^{i j}
\end{aligned}
$$

\footnotetext{
${ }^{1}$ In planar constraint, one of the eigenvalues is zero, which makes the determinant equal to zero.

${ }^{2}$ The factor of 2 is just here for a convenience purpose.
} 
One may define an effective viscosity $\eta_{\text {eff }}=\eta_{0} \alpha^{\frac{n-1}{2}}$ and introduce the Ostwald vector $\vec{O}$ whose contravariant $i$-component is $O^{i}=\frac{1}{\alpha} D_{j}(\alpha) S^{i j}=$ $D_{j}(\ln \alpha) S^{i j}$. The generalized Navier-Stokes equation for incompressible flows is then:

$$
\rho\left(\frac{D \vec{v}}{D t}\right)=\vec{f}_{V}-G \overrightarrow{r a d} p+\eta_{e f f} \Delta \vec{v}+(n-1) \eta_{e f f} \vec{O}
$$

where $\vec{f}_{V}$ are the forces per unit volume. One can easily check that, setting $n=1$ (which corresponds to the Newtonian case) in the above equation (5), the regular Navier-Stokes equation for incompressible flows is recovered. Note that, written as it is, equation (5) is automatically covariant.

\section{Application to droplet spreading}

\subsection{Evolution of the spreading radius: the Newtonian case}

In order to study the spreading of a non-Newtonian droplet, a preliminary step consists in considering the Newtonian case. Next, the generalization to non-Newtonian fluids can be done. Figure 1 displays the notations used in

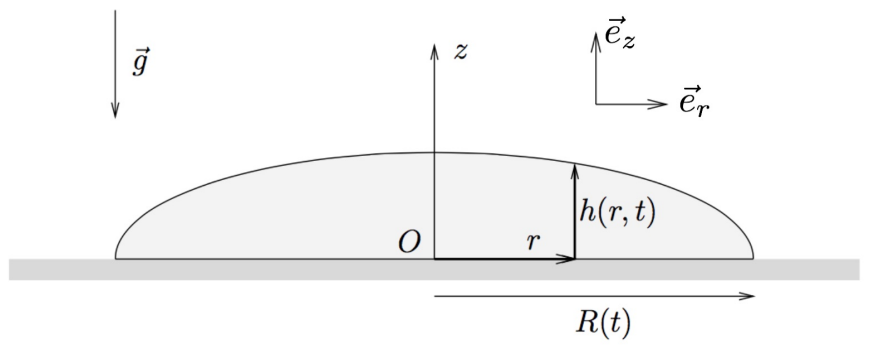

Figure 1. Spreading of an axisymmetric droplet in the $z=0$ plane.

the following. We will assume hereafter that the lubrication approximation applies, i.e. vertical length scale small relative to radial length scale, incompressible flow, low Reynolds number, and negligible surface tension effects. As such, we model the droplet's flow as a gravity current. The Navier-Stokes equation is given by:

$$
\rho \frac{D \vec{v}}{D t}=\rho\left(\partial_{t} \vec{v}+(\vec{v} \cdot g r \overrightarrow{r a d}) \vec{v}\right)=-g \overrightarrow{r a} d p+\eta \Delta \vec{v}+\rho \vec{g}
$$

The projection of (6) on the $r$ and $z$ axis gives, with $\nu=\frac{\eta}{\rho}$ :

$$
\left\{\begin{array}{c}
\partial_{t} v_{r}+v_{r} \partial_{r} v_{r}+v_{z} \partial_{z} v_{r}=-\frac{1}{\rho} \partial_{r} p+\nu\left(\partial_{r r}^{2} v_{r}+\frac{1}{r} \partial_{r} v_{r}-\frac{v_{r}}{r^{2}}+\partial_{z z}^{2} v_{r}\right) \\
\partial_{t} v_{z}+v_{r} \partial_{r} v_{z}+v_{z} \partial_{z} v_{z}=-\frac{1}{\rho} \partial_{z} p+\nu\left(\partial_{r r}^{2} v_{z}+\frac{1}{r} \partial_{r} v_{z}+\partial_{z z}^{2} v_{z}\right)-g
\end{array}\right.
$$

For $R e=\frac{v_{r} R}{\nu} \ll 1$, the equation system (7) simplifies to:

$$
\left\{\begin{array}{c}
\frac{1}{\rho} \partial_{r} p=\nu \partial_{z z}^{2} v_{r} \\
\frac{1}{\rho} \partial_{z} p=\nu \partial_{z z}^{2} v_{z}-g
\end{array}\right.
$$


Let us introduce the function $f(r, z, t)=z-h(r, t)$. For a fluid particle at the air-liquid interface, we have by definition $f=0$. Now the fluid particle is a Lagrangian invariant, thus $\frac{D f}{D t}=0$. Since $\frac{D f}{D t}=v_{z}-\partial_{t} h-v_{r}(r, z=$ $h, t) \partial_{r} h=0$, we have:

$$
v_{z}=\partial_{t} h+v_{r}(r, z=h(r, t), t) \partial_{r} h
$$

One integrates the continuity equation over the thickness of the droplet. The continuity equation is, $\rho$ being understood as a function $\rho(r, z, t)$ :

$$
\partial_{t} \rho+\frac{1}{r} \partial_{r}\left(\rho r v_{r}\right)+\partial_{z}\left(\rho v_{z}\right)=0
$$

with $\rho(r, z, t)=\rho \Theta(h(r, t)-z)$ where $\Theta$ is the Heaviside function. Because $\partial_{t} \rho=\rho \delta(h(r, t)-z) \partial_{t} h, \int_{0}^{\infty} d z \partial_{t} \rho=\rho \partial_{t} h \int_{0}^{\infty} d z \delta(h-z)=\rho \partial_{t} h$ and $\int_{0}^{\infty} d z \partial_{z}\left(\rho v_{z}\right)=\left(\rho v_{z}\right)_{\infty}-(\rho Z)_{0}=0$ because $\rho_{\infty}=0$ and $\left(v_{z}\right)_{0}=0$ Therefore from (10) one obtains:

$$
\partial_{t} h+\frac{1}{r} \partial_{r}\left(r \int_{0}^{h(r, t)} d z v_{r}(r, z, t)\right)=0
$$

If one assumes that the viscosity term can be neglected in the $z$-projection of the simplified Navier-Stokes equation (8), then the pressure follows an hydrostatic law given by:

$$
p(r, z, t)=p_{0}-\rho g(z-h(r, t)),
$$

where $p_{0}$ standing for the atmospheric pressure. If one integrates the $x$ projection of the simplified Navier-Stokes equation (8) with the boundary conditions : $v_{r}(r, z=0, t)=0$ (no-slip) and $\left.\partial_{z} v_{r}\right)_{z=h}=0$ (no-shear), one finds:

$$
v_{r}(r, z, t)=\frac{g}{\nu} z\left(\frac{z}{2}-h\right) \partial_{r} h
$$

Using equations (11) and (13), one finds

$$
\partial_{t} h=\frac{g}{3 \nu} \frac{1}{r} \partial_{r}\left(r h^{3} \partial_{r} h\right)
$$

The latter equation, in its present form, cannot be solved analytically. Nevertheless, the geometry considered suggests to look for a self-similar solution (see [3] and [7]) of the form $h(r, t)=H(t) f(X)$, where $X=\frac{r}{R(t)}$, and to insert it in (14). The droplet volume $\Omega$ is given by:

$$
\Omega=b R^{2}(t) H(t), \text { where the constant } b=2 \pi \int_{0}^{1} X d X f(X)
$$

The volume conservation yields:

$$
R \frac{d H}{d t}+2 H \frac{d R}{d t}=0
$$


Let us evaluate the right-hand side of equation (14) when $r$ goes to 0 :

$$
\begin{aligned}
\frac{1}{r} \partial_{r}\left(r h^{3} \partial_{r} h\right) & =\frac{H^{4}(t)}{R^{2}(t)} \frac{1}{X} \frac{d}{d X}\left(X f^{3}(X) f^{\prime}(X)\right) \\
& =\frac{H^{4}(t)}{R^{2}(t)} \frac{1}{X}\left(f^{3}(X) f^{\prime}(X)+3 X f^{2}(X) f^{\prime 2}(X)+X f^{3}(X) f^{\prime \prime}(X)\right)
\end{aligned}
$$

As $\left.\partial_{r} h\right)_{r=0}=0$, we have $f^{\prime}(0)=0$, so a Taylor expansion for small $X$ gives $f^{\prime}(X)=X f^{\prime \prime}(0)$. Thus $\lim _{r \rightarrow 0} \frac{1}{r} \partial_{r}\left(r h^{3} \partial_{r} h\right)=\frac{H^{4}(t)}{R^{2}(t)} 2 f^{3}(0) f^{\prime \prime}(0)$. Because the curvature radius is negative in 0 , one can rewrite the latter, using (14), as:

$$
\dot{H}(t)=-C \frac{H^{4}(t)}{R^{2}(t)},
$$

where $\mathrm{C}$ is a constant. By eliminating $H(t)$ and $\dot{H}(t)$ in equation (16) by using equations (17) and (15). One finds a differential equation for $R(t)$ :

$$
\dot{R} R^{7}=\frac{1}{2} \frac{C \Omega^{3}}{b^{3}}
$$

that we integrate in:

$$
R^{8}(t)=\frac{4 C \Omega^{3}}{b^{3}} t+R^{8}(0)
$$

As soon as one can neglect $R(0)$ in front of $R(t)$, the radius spreading law is thus in $t^{1 / 8} 3$.

\subsection{Evolution of the spreading radius: the power-law case}

Now that a method to study the droplet spreading has been established for Newtonian fluids, let us apply it to non-Newtonian fluids and emphasize the differences. Considering the geometry of the experiment and the same approximations as previously enables to calculate $\alpha$, and consequently $\eta_{\text {eff }}$ and $\vec{O}$; neglecting non dominant terms in the sum, one finds:

$$
\vec{O}=\left(\begin{array}{c}
\eta_{e f f}=\eta_{0}\left(\tau \partial_{z} v_{r}\right)^{n-1} \\
\partial_{z z}^{2} v_{r} \\
0 \\
\frac{1}{\partial_{z} v_{r}}\left(\partial_{z} v_{r} \partial_{r z}^{2} v_{r}+2 \partial_{z z}^{2} v_{r} \partial_{z} v_{z}\right)
\end{array}\right)
$$

The simplified generalized Navier-Stokes equation (5) is then

$$
\left\{\begin{array}{c}
\partial_{r} p=\frac{\eta_{0}}{\tau} \partial_{z}\left(\left(\tau \partial_{z} v_{r}\right)^{n}\right) \\
\partial_{z} p=-\rho g
\end{array}\right.
$$

We obtain that the pressure still follows a hydrostatic law and that the radial velocity field is

$$
v_{r}(r, z, t)=\frac{h}{\tau} \frac{n}{n+1}\left(\frac{\tau \rho g h\left(-\partial_{r} h\right)}{\eta_{0}}\right)^{1 / n}\left(1-\left(1-\frac{z}{h}\right)^{\frac{n+1}{n}}\right)
$$

\footnotetext{
${ }^{3}$ Note the difference with the well known Tanner law for which the surface tension is not negligible and leads to an evolution law in $t^{1 / 10}$. [12]
} 
(Note that setting $n=1$ the equation (13) is recovered.) As in the Newtonian case, the integration of the continuity equation over the droplet height gives

$$
\partial_{t} h+\frac{1}{r} \partial_{r}\left(r \int_{0}^{h(r, t)} v_{r}(r, z, t) d z\right)=0
$$

Using (23), one finally finds the differential equation in $h$

$$
\partial_{t} h+\frac{1}{r} \partial_{r}\left(r \frac{h^{2}}{\tau} \frac{n}{2 n+1}\left(\frac{\tau \rho g h\left(-\partial_{r} h\right)}{\eta_{0}}\right)^{1 / n}\right)=0
$$

In the Newtonian case, the self-similar solution considered is $h(r, t)=H(t) f(X)$, where $X=\frac{r}{R(t)}$. In the non-Newtonian case the latter no longer works as it raises divergence when injected in (25). We find a new similarity solution by looking at a self-similar variable with an exponent: $h(r, t)=H(t) f(X)$, where $X=\left(\frac{r}{R(t)}\right)^{\beta}$. We adjusted the value of $\beta$ to eliminate the divergence when $\mathrm{r}$ goes to 0 . Only one value is acceptable, $\beta=\frac{n(n+1)}{2}$. The solution is then

$$
R(t)^{\frac{3 n+5}{n}}=\lambda(n, K) t+R(0)^{\frac{3 n+5}{n}}
$$

As soon as we can assume $R(t) \gg R(0)$, the radius follows a power law $R(t) \approx t^{\frac{n}{3 n+5}}$. This behaviour is similar to the one reported in [16]. Thanks to equation (26), it is possible to find out $n$ from the spread radius of the gravity current. It is unfortunately difficult to find out the value of $K$. Indeed the function $\lambda(n, K)$ depends on $\mathrm{f}(0)$ whose value is unknown.

\section{Recovering the spreading laws though dimensional analysis}

In the previous section the spreading laws have been derived exactly. Nevertheless there exists an alternative method, based on dimensional analysis, to quickly recover the expected behaviours. One should keep in mind that the analysis that follows is just dimensional, done with hands, and does not constitute a formal demonstration. The idea is just to write what physically happens, in our case, when the droplet spreads. It is a competition between pressure forces that make the droplet spread and viscous forces that resist its motion. Writing the balance gives:

$$
\partial_{r} p \approx \eta \triangle v
$$

We will evaluate this balance considering characteristic lengths and time. The vertical length is referred to $H$ and the horizontal one is referred to $R$. The self-similar hypothesis also enables to write a relation between the two characteristic lengths : $\Omega \approx R^{2} H=c s t$. Since the pressure is hydrostatic, one has $p \approx \rho g H$.

For the Newtonian case, inserting characteristic dimensions, equation (27) becomes:

$$
\rho g \frac{H}{R} \approx \eta \frac{1}{H^{2}} \frac{R}{t}
$$


Substituting $H$ by $\frac{\Omega}{R^{2}}$, one finds the law $\frac{\rho g}{\eta} t \Omega^{3} \approx R^{8}$, which is the expected behaviour.

For the non-Newtonian case we also have to make explicit the dependence of $\eta$ on the characteristic dimensions. We write $\eta_{e f f} \approx K\left(\partial_{z} v_{r}\right)^{n-1} \partial_{z z}^{2} v_{r} \approx$ $K\left(\frac{1}{H} \frac{R}{t}\right)^{n-1} \frac{R}{t} \frac{1}{H^{2}}$. Inserting it in equation (27), we obtain:

$$
\rho g \frac{H}{R} \approx K\left(\frac{1}{H} \frac{R}{t}\right)^{n-1} \frac{R}{t} \frac{1}{H^{2}}
$$

Substituting $H$ by $\frac{\Omega}{R^{2}}$ again, ones find the law $\frac{\rho g}{K} \Omega^{2+n} t^{n} \approx R^{3 n+5}$, which is also the expected behaviour.

\section{Theoretical expressions applied to experimentally found viscous parameters}

\subsection{Materials and methods}

In order to complete the theoretical study presented before, we performed some experiments with viscous and non-Newtonian fluids (glycerol and silicon oils). We emphasize here that our aim is here to investigate how the theoretical expressions derived can be applied to experimental measures of non-Newtonian fluids viscosity. It only is a proof of principle with no metrology pretence at this stage.

We worked with viscosities ranging from $10^{-1}$ to $10^{4}$ Pa.s. Temperature fluctuations around the working temperature of $20^{\circ} \mathrm{C}$ were neglected as the viscosities of the studied fluids undergo negligible variations around $20^{\circ} \mathrm{C}$. We studied droplet spreading dominated by gravity effects. Initially contained in a cylinder (its diameter is $73 \mathrm{~mm}$ ), the fluid is released when one removes the cylinder: it is then free to spread. The droplet radius is monitored thanks to a camera and a Matlab code which enables to track automatically the radius on the set of pictures. See Figure 2.

\subsection{Results}

According to equation (26), the value of $n$ should not depend on the volume of the droplet. For this reason, we tested different volumes: $\mathrm{V}=76 \mathrm{ml}$ $(\mathrm{h}=\mathrm{D} / 4), \mathrm{V}=51 \mathrm{ml}(\mathrm{h}=\mathrm{D} / 6), \mathrm{V}=38 \mathrm{ml}(\mathrm{h}=\mathrm{D} / 8)$.

In order to extract $n$ from the curves, we plotted $\log (\mathrm{R})$ versus $\log (\mathrm{t})$. Measuring the line slope, expected to be equal to $\frac{n}{3 n+5}$, it is possible to extract $n$. It appears, as expected according to formula (26), that the values of $n$ do not depend on the volume of the droplet. We found one slope for glycerol (leading to $n \approx 0.3$ ) spreading. All the silicone oil samples showed a more complex behavior: a two-slope spreading (see Figure 3 and section 5.3 for more explanations on the values and a more detailed uncertainty treatment).

In order to evaluate more quantitatively the two slope behavior, we use a matlab code for linear regressions. We did two linear regressions with a floating junction point. The value of this point is then fixed as the one which minimizes errors corresponding to the two linear regressions. 

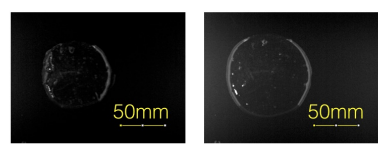

$\mathrm{t}=120 \mathrm{~s}$

$\mathrm{t}=540 \mathrm{~s}$

$\mathrm{R}=39.8 \mathrm{~mm} R=45.2 \mathrm{~mm}$
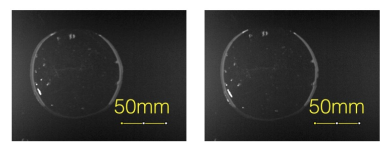

$t=1710 \mathrm{~s} \quad \mathrm{t}=2670 \mathrm{~s}$

$\mathrm{R}=52 \mathrm{~mm} \quad \mathrm{R}=54.7 \mathrm{~mm}$

(a) Four pictures during the speading

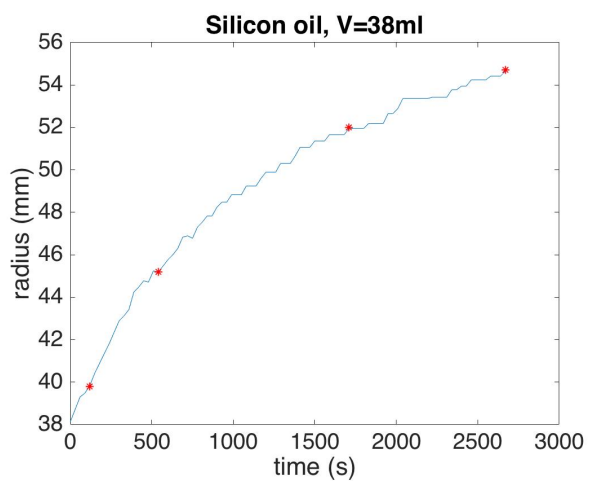

(b) Global graph of evolution, stars refer to the previous pictured data.

FiguRE 2. Evolution of the droplet radius of Silicon oil when released from the cylinder. The volume of the droplet is 38 $m l$.

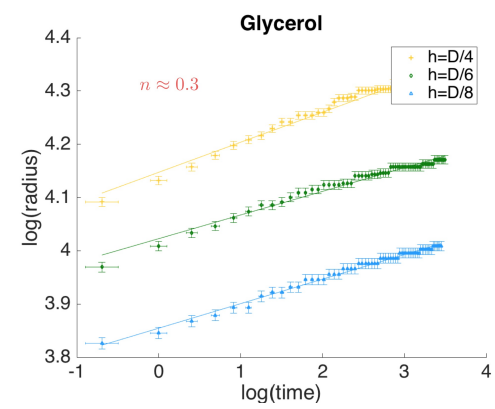

(a) Only one slope yielding $n \approx 0.3$.

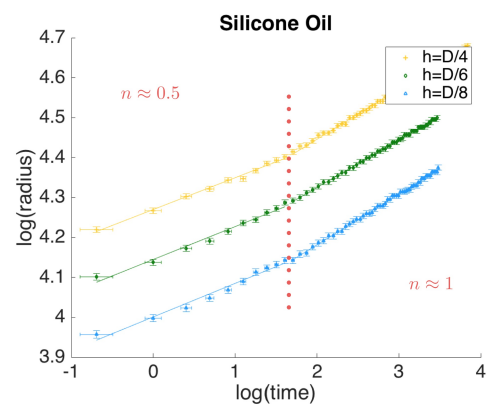

(b) Two slopes: $n \approx 0.5$ at short times and $n \approx 1$ at long times.

FiguRE 3. Different behaviors for the glycerol and the silicon oil, the value of the slope(s) leads to $n$.

\subsection{Discussion}

In order to correctly derive $n$ from the experimental data, one should be careful. Glancing indeed at Figure 4, one can notice that in the region of interest (the slope belonging to $[0 ; 1 / 3]$ ) the evolution of $\mathrm{n}$ is very quick. This makes the $n$ extrapolation very sensitive.

However because the image treatment can be achieved very precisely (up to $0.1 \mathrm{~mm}$ ) the main uncertainty source comes from the slope determination. Let's take an example (from measured data) to estimate the uncertainty on $n$ one can expect. 


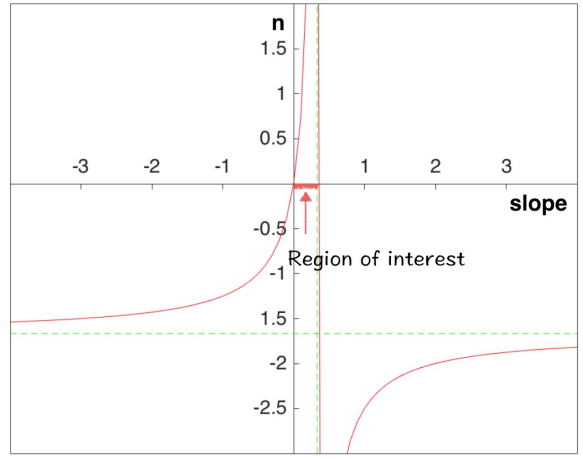

FIGURE 4. Evolution of $n$ with the measured slope. In the region of interest the variation is quick, which makes the extrapolation sensitive.

Because of the linear regression we get for one silicon oil measurement $(h=D / 4)$, in the second line regression, slope $=(0.13 \pm 0.08) 10^{-3} \mathrm{~m} . \mathrm{s}^{-1}$. The $\mathrm{n}$ coefficient is then given by $n=\frac{-5 \times \text { slope }}{3 \times \text { slope }-1}$. The uncertainty propagation gives us : $n=0.9 \pm 0.2$. With this numerical illustration, one can see how precision degrades. This is a consequence of the slope determination which is difficult. Another source of inaccuracy arises when one determines $n$ from the slope. In the example above, only one or two significant digits accuracy could be achieved. A careful manipulation (yielding more precise measures) should enable to improve precision on $n$ value.

Let's consider the two slopes behavior. One could argue that the reason for the existence of two slopes is the following: at the beginning of the experiment, the radius is not large enough in front of the initial radius, which should prevent the radius from following the $t^{\frac{n}{3 n+5}}$ law. But the fact that the fit works for the glycerol solutions weakens this argument.

The change in the value of $n$ looks like a time-dependent non-Newtonian fluid behavior, akin to thixotropy. A fluid is said to be thixotropic fluid when under a constant speed constraint, it experiences a decrease in viscosity with time [4]. This behavior is explained by a structural evolution of the fluid (in fact a de-structuration). This behavior fits very well silicone oils whose helical structure enables them to stagger. Moreover thixotropic behavior already have been observed in silicon oil [10,9]. Thus one can interpret the first slope as the re-arrangement of the polymer chains with one another and the second slope as the spreading with re-arranged polymer chains. This second step lasts at least for five hours (duration of the longest experiment we did with it).

The explanation of why the thixotropic behavior only appears (at the monitored time-scale) for silicon oils is that its structure reorganization time 
fits with the experiment's duration. Considering the glycerol, its short molecular length should enable an instantaneous reorganization.

\section{Conclusion}

Non-Newtonian fluids are often described with the Ostwald power-law. We showed here that considering this power-law behavior and working in a covariant formalism (all the more convenient because it is independent of any choice of coordinate system), one can reach a generalized form of NavierStokes equation. The Ostwald vector, which is added to the Navier-Stokes equation to extend it to non-Newtonian fluids, is characterized by the Ostwald power-law exponent $n$. Solving the generalized Navier-Stokes equation in cylindrical coordinates enables one to recover the well known spreading law $R \propto t^{\frac{n}{3 n+5}}$. It is interesting to note that this law can be recovered through dimensional analysis too, a fact which does not appear to have been reported in the literature before.

An interesting application of this spreading law is that it could enable the estimation of the power law exponent $n$ from fluid spreading experiments. Experiments and an order of magnitude analysis show that this method has an important limitation since very precise measurements are required in order recover sufficiently accurate values of $n$. Further work is needed to confirm the potential of this method which could be very valuable for flows of materials which are not accessible or impossible to handle such as lava flows.

Acknowledgements The authors wish to thank Etienne Jaupart for useful discussions related to the derivation of the scaling laws.

\section{References}

1. Gulraiz Ahmed, Mathieu Sellier, Yeaw Chu Lee, Mark Jermy, and Michael Taylor, Modeling the spreading and sliding of power-law droplets, Colloids and Surfaces A: Physicochemical and Engineering Aspects 432 (2013), 2-7.

2. NJ Balmforth, RV Craster, P Perona, AC Rust, and R Sassi, Viscoplastic dam breaks and the bostwick consistometer, Journal of non-newtonian fluid mechanics 142 (2007), no. 1-3, 63-78.

3. Grigory Isaakovich Barenblatt, Scaling, self-similarity, and intermediate asymptotics, Cambridge University press, 1996.

4. H.B. Barnes, Thixotropy - a review, Journal of Non-Newtonian Fluid Mechanics 70 (1997), 1-33.

5. Martin Devaud and Thierry Hocquet, Lagrangian sound, Hal.

6. JJ Foit, Spreading under variable viscosity and time-dependent boundary conditions: estimate of viscosity from spreading experiments, Nuclear engineering and design 227 (2004), no. 2, 239-253. 
7. Julio Gratton, Fernando Minotti, and Swadesh M Mahajan, Theory of creeping gravity currents of a non-newtonian liquid, Physical Review E 60 (1999), no. 6, 6960.

8. Étienne Guyon, Jean-Pierre Hulin, and Luc Petit, Hydrodynamique physique, CNRS Editions, 2001.

9. S.J. Hahn, T. Ree, and H. Eyring, Flow mechanism of thixotropic substances, Journal of the American Chemical Society (1959).

10. Z. Kökuti, J. Kokavecz, A. Czirjak, I. Holczer, A. Danyi, Z. Gabor, G. Szabo, N. Pezsa, P. Ailer, and L. Palkovics, Nonlinear viscoelasticity and thixotropy of a silicon fluid, International journal of engineering IX (2011).

11. Sandro Longo, Vittorio Di Federico, Renata Archetti, Luca Chiapponi, Valentina Ciriello, and Marius Ungarish, On the axisymmetric spreading of non-newtonian power-law gravity currents of time-dependent volume: an experimental and theoretical investigation focused on the inference of rheological parameters, Journal of Non-Newtonian Fluid Mechanics 201 (2013), 69-79.

12. S. Mechkov, A.M. Cazabat, and G. Oshanin, Post-tanner stages of droplet spreading: the energy balance approach revisited, Journal of Physics: Condensed Matter 21 (2009), no. 464131.

13. J.-M. Piau, Consistency slump and spreading tests: Practical comments, Journal of Non-Newtonian Fluid Mechanics 135 (2006), no. 177-178.

14. J-M Piau and K Debiane, Consistometers rheometry of power-law viscous fluids, Journal of non-newtonian fluid mechanics 127 (2005), no. 2-3, 213-224.

15. Salima Rafaï, Daniel Bonn, and Arezki Boudaoud, Spreading of non-newtonian fluids on hydrophilic surfaces, Journal of Fluid Mechanics 513 (2004), 77-85.

16. R.Sayag and M.G. Worster, Axisymmetric gravity currents of power-law fluids over a rigid horizontal surface, JMF Rapids (2012).

17. W.R. Schowalter, Mechanics of non-newtonian fluids, Princeton University, 1978.

18. M Sellier, JW Grayson, L Renbaum-Wolff, M Song, and AK Bertram, Estimating the viscosity of a highly viscous liquid droplet through the relaxation time of a dry spot, Journal of Rheology 59 (2015), no. 3, 733-750.

19. VM Starov, AN Tyatyushkin, MG Velarde, and SA Zhdanov, Spreading of nonnewtonian liquids over solid substrates, Journal of colloid and interface science 257 (2003), no. 2, 284-290.

20. LH Tanner, The spreading of silicone oil drops on horizontal surfaces, Journal of Physics D: Applied Physics 12 (1979), no. 9, 1473.

21. AS Uppal, RV Craster, and OK Matar, Dynamics of spreading thixotropic droplets, Journal of Non-Newtonian Fluid Mechanics 240 (2017), 1-14.

L. Devaud

e-mail: louisiane.devaud@ens-lyon.fr

École Normale Supérieure de Lyon

M. Sellier

e-mail: mathieu.sellier@canterbury.ac.nz

Department of Mechanical Engineering

University of Canterbury 
L. Devaud, M. Sellier and A.-R. Al-Behadili

\author{
A.-R. Al-Behadili \\ Department of Mechanical Engineering \\ University of Canterbury
}

